\title{
Retinal organoids on-a-chip: a 3D printed micro-millifluidic bioreactor for long-term retinal organoid maintenance
}

\author{
Authors: \\ Yuntian Xue ${ }^{1}$, Magdalene J. Seiler, ${ }^{4,2,7}$, William C. Tang ${ }^{1}$, Jasmine Y. Wang ${ }^{6}$, Andrew W. \\ Browne $e^{1,3,5}$
}

Affiliation:

1. University of California, Irvine; Biomedical Engineering; Irvine CA

2. University of California, Irvine; Stem Cell Research Center; Irvine CA

3. University of California, Irvine, Gavin Herbert Eye Institute Department of Ophthalmology; Irvine CA

4. University of California, Irvine; Physical Medicine \& Rehabilitation; Irvine, CA

5. University of California, Irvine; Institute for Clinical and Translational Science; Irvine, CA

6. University of California, Irvine; School of Biological Sciences; Irvine, CA

7. University of California, Irvine, Anatomy \& Neurobiology, Irvine, CA

\section{Corresponding Author:}

Andrew W. Browne, MD, PhD, abrowne1@hs.uci.edu

\section{Grant support:}

California Institute of Regenerative Medicine (CIRM) grant number TRAN1-10995

RPB unrestricted grant to UCI Department of Ophthalmology

ICTS KL2 Grant number is KL2 TR001416 


\section{ABSTRACT:}

Retinal degeneration is a leading cause of vision impairment and blindness worldwide and medical care for advanced disease does not exist. Stem cell-derived retinal organoids (RtOgs) became an emerging tool for tissue replacement therapy. However, existing RtOg production methods are highly heterogeneous. Controlled and predictable methodology and tools are needed to standardize RtOg production and maintenance. In this study, we designed a shear stress-free micro-millifluidic bioreactor. We used a stereolithography (SLA) 3D printer to fabricate a mold from which Polydimethylsiloxane (PDMS) was cast. The multi-chamber bioreactor design and fabrications methods easily combined micro and millimeter features with very low cost and short manufacturing time. We optimized the chip design using in silico simulations and in vitro evaluation to optimize mass transfer efficiency and concentration uniformity in each culture chamber. We successfully cultured RtOgs on an optimized bioreactor chip for 37 days. We also characterized the RtOgs produced by static dish culture and chip culture methods using qualitative and quantitative techniques. Phase contrast imaging showed that both conventional and chip-cultured RtOgs developed a transparent outermost surface structure. Fluorescence lifetime imaging (FLIM) showed that RtOgs on the chip had significantly lower long lifetime species (LLS) ratio than static cultured ones, which demonstrated that bioreactor cultured RtOgs exhibited less oxidative stress. RtOgs in bioreactor culture demonstrated higher NADH signal overall, but both bioreactor and conventional cultures showed similar free/bound NADH ratio over time, which indicated normal differentiation time course. RtOg gene expression was examined by fluorescence imaging and quantitative polymerase chain reaction (qPCR) analyses. RtOgs in both groups showed thick nuclear outer layers expressing CRX on day 120 of differentiation. The gene profiling showed both groups expressed retinal progenitor genes and most of the tested photoreceptor markers. We, therefore, validated an autonomous micro-millifluidic device with significantly reduced shear stress and lower oxidative stress to produce RtOgs of equal or greater quality than those maintained in conventional static culture. 
Key words: Retinal organoids; Microfluidics; Millifluidics; 3D printing; Fluorescence lifetime imaging; Phasor approach; Functional imaging. 
INTRODUCTION:

Retinal degeneration (RD) is a leading cause of vision impairment and blindness worldwide.

3 Visual degeneration can originate in any of the cell types in the retina. Some of the more common

4 visual degenerations stemmed from death and/or dysfunction of the photoreceptors (PR) and

5 retinal pigmented epithelial (RPE) cells. These irreversible cell damages lead to vision loss

6 manifested in age-related macular degeneration (AMD) and retinitis pigmentosa (RP). Retinal

7 sheets and dissociated retinal cells are candidates for retinal tissue replacement therapy.

8 However, both tissue sources have inherent limitations. Historically, retinal sheets derived from

9 fetal neurosensory retina and RPE transplanted into the subretinal space demonstrated utility to

10 restore vision and neurosensory functions ${ }^{1-7}$ in animals ${ }^{8-10}$ and humans ${ }^{11}$. However, the use of

11 fetal tissue carried complex social, ethical, and political implications. Transplantation of

12 dissociated photoreceptor precursors overcame the ethical issues and demonstrated some visual

13 function improvements ${ }^{12,13}$ but dissociated cells introduced additional challenges ${ }^{14-17}$ that mainly

14 resulted from insufficient cell type differentiation, polarization and eventual cell death.

15 With the advent of human embryonic (hESCs) ${ }^{18}$ and induced pluripotent stem cells (iPSCs)

$16{ }^{19}$, stem cell-derived retinal organoids (RtOgs) have emerged as tools that exhibit the combined

17 advantages of retinal sheets and differentiated retinal cells. RtOgs are 3D spheroid tissues that

18 self-organized into layered retinal tissues containing retinal ganglion cells, rods and cones ${ }^{20-22}$.

19 Transplantation of RtOgs have been shown to restore vision in retina degenerated rats ${ }^{23}$, mouse

$20{ }^{24}$ and primate ${ }^{25}$ models with RD. Even so, the current state-of-the-art RtOg production methods

21 are highly heterogeneous due to their use of different cell lines, culturing times, culturing methods,

22 manual labor and imprecise tissue selections ${ }^{26}$. A comparative study revealed that RtOgs

23 differentiated from iPSCs showed stage specific, cell line and methodological differences ${ }^{27}$. This

24 heterogeneity and imprecision limit human RtOg procurement for preclinical trials ${ }^{26}$ and in vitro

25 investigations. Many approaches, including bioreactors ${ }^{28-34}$ and optimized production protocols

2626,35 are investigated to standardize RtOg production and maintenance over months. Controlled 
27 and predictable RtOg production is important to ensure a quality-controlled tissue product that is 28 suitable for transplantation.

29 In recent years, many in vitro cell culture platforms have emerged for organoid differentiation 30 and maintenance at the macro-, milli-, and microscales. Macro-scaled platforms are typically 31 utilized for their ease and effectiveness in producing organoids, while milli-scaled systems ( $\geq 1$ $32 \mathrm{~mm}$ ) are employed for relatively high flow rates, cell-cell interaction, and less frequent media 33 changes and thus less organoid perturbation and lower probability for damage ${ }^{29}$. Considering the 34 costs associated with the relatively high media volumes required by the macro-scaled bioreactors, 35 microscale devices $(<100 \mu \mathrm{m})$ are steadily growing in popularity ${ }^{36}$. Microfluidic devices shared 36 the advantages of millifluidic devices, with the further advantage of even lower media 37 consumption. However, the dimensional limits of traditionally fabricated microfluidics devices 38 hinder its application to organoids research since organoids are 3D spherical tissues that can 39 grow up to several millimeters in size. Fig. 1 summarizes current published organoid bioreactors 40 and their advantages and disadvantages. The integration of micro- and millifluidic device is a 41 promising solution for organoids differentiation and maintenance. 


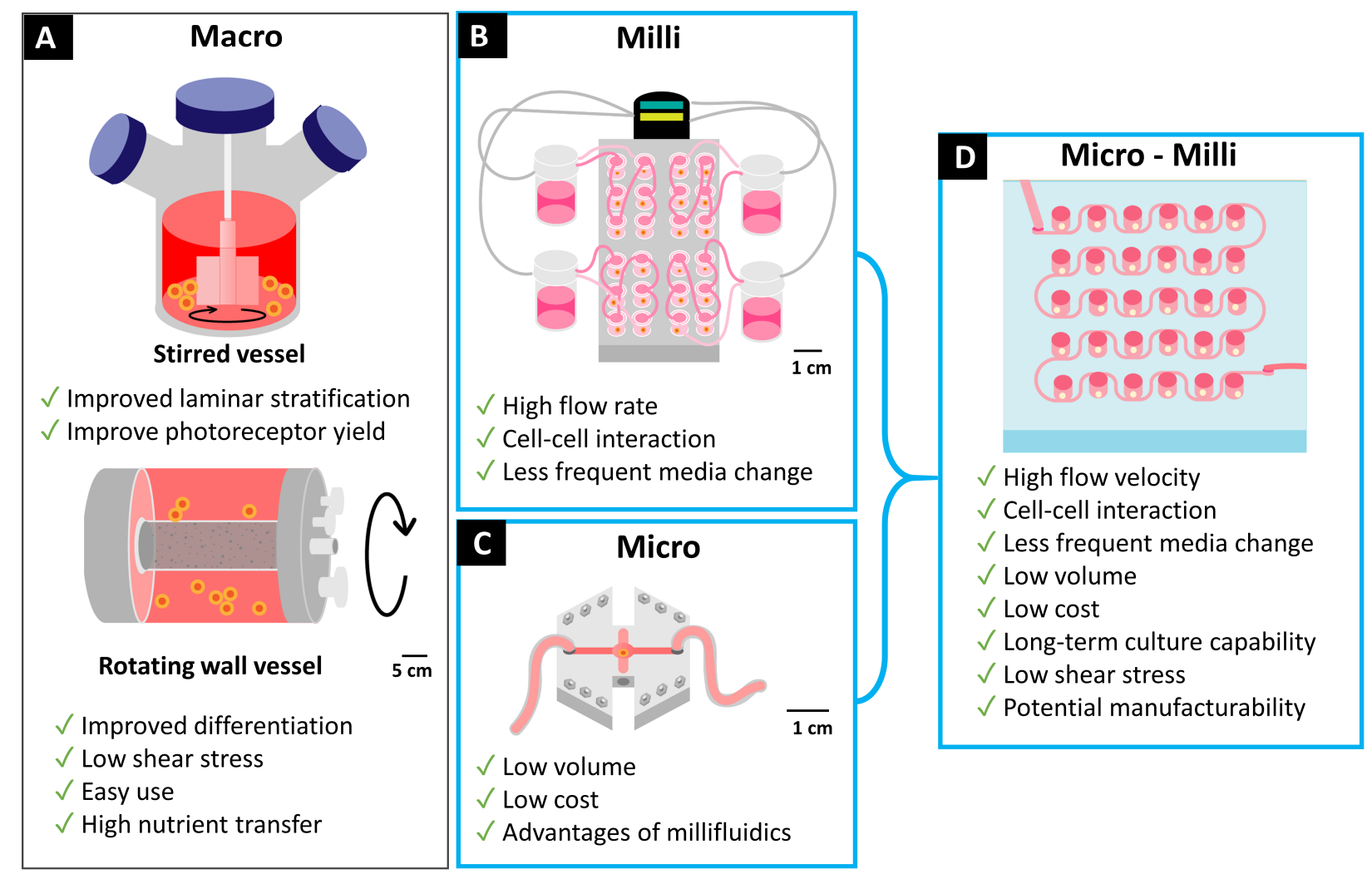

Figure 1: Review of Organoid Bioreactors. (A) Macro scale bioreactors: stirred/spinning and rotating wall vessels ${ }^{37} ;(B)$ (B) Millifluidic bioreactor 38 ; (C) Microfluidic bioreactor 39 ; (D) Micromillifluidic bioreactor in this article.

In this study, we designed and fabricated a shear stress-free micro-millifluidic bioreactor for

47 use in RtOg culture and maintenance. We used a high resolution (25 $\mu \mathrm{m})$ stereolithography (SLA) 3D printer to fabricate the mold for Polydimethylsiloxane (PDMS) molding, which easily combined micro and millimeter features in one design with very low cost and short manufacturing time. First, we simulated the fluidic design parameters in COMSOL to optimize the fluidic transports in the

51 chip design. We evaluated 3 different factors that could affect mass transfer efficiency and 52 uniformity. We then successfully cultured RtOgs on the designed chip platform for more than one 53 month (37 days). Finally, we did a comparative study to characterize the RtOgs produced by dish 54 culture (denoted as "static" because there was no constant media flow through the dish) and chip 55 culture. We compared live organoids both qualitatively and quantitatively. 


\section{METHODS}

\section{$58 \quad$ COMSOL simulation}

The simulation was performed using finite element analysis software, COMSOL Multiphysics (COMSOL, Inc, Palo Alto, CA, USA). COMSOL was used to evaluate different chip designs and

61 flow channel configurations to optimize mass-transport dynamics in culture chambers with 62 different heights. Three major factors that affected the mass transfer rate were taken into 63 consideration: 1) channel width (1000 or $500 \mu \mathrm{m}$ wide), 2) channel configuration relative to culture 64 chambers (linear single-sided chambers, serpentine alternating side chambers, serpentine with 65 integrated mixer) and 3) the culture chamber height (2 or $4 \mathrm{~mm}$ tall).

The simulation parameters are listed in Table 1. The initial concentration of the whole system was set to zero, which was considered the most extreme condition. The left end of the channel was set as the inlet with concentration of $1 \mathrm{~mol} / \mathrm{m}^{3}$ as the boundary condition.

Table 1: Simulation parameters

\begin{tabular}{|c|c|}
\hline Physics & Laminar flow \& Transport of diluted species \\
\hline Study type & Time dependent \\
\hline Material & Water \\
\hline Diffusion coefficient $\left(\mathbf{m}^{2} / \mathbf{s}\right)$ & $6.00 E-10$ \\
\hline Boundary conditions $\left(\mathbf{m o l}^{\mathbf{m}} \mathbf{m}^{\mathbf{3}}\right)$ & Cinitial $=0$, Cinlet $=1$ \\
\hline
\end{tabular}

71

\section{Chip design and fabrication}

73 The mold was designed using SolidWorks (SolidWorks Corp., Waltham, MA, USA) and the

74 final design used for RtOg culture had the dimensions shown in Fig. 2A with channel height of

$75200 \mu \mathrm{m}$ and chamber height of $2 \mathrm{~mm}$. The chambers were arranged in a $6 \times 5$ array with the 
distance between each chamber at $9 \mathrm{~mm}$, which was the same as that of a 96-well plate for

77 compatibility with subsequent imaging steps. The mold was produced with $25 \mu \mathrm{m}$ resolution with

78 the Formlabs Form 3B printer (Formlabs, Somerville, MA, USA) using standard clear resin

79 (Formlabs) (Fig. 2B). After printing, the mold was cleaned with $90 \%$ isopropanol to remove any

80 resin residue. The mold was then air dried for 24 hours and cured with ultraviolet light for 30

81 minutes.

82 The bioreactor was fabricated from the printed mold similar to the molding steps in soft 83 lithography ${ }^{40}$. Polydimethylsiloxane (PDMS) Sylgard 184 (Dow Corning, Midland, MI, USA) was

84 mixed manually for 10 minutes at a 10:1 ratio (base elastomer/curing agent). After degassing in

85 a vacuum chamber, the PDMS was poured over the 3D-printed mold until the level reached the

86 top of the culture chamber features and degassed again in a vacuum desiccator to remove

87 bubbles (Fig. 2C). After 48 hours of curing under room temperature, the molded PDMS piece was

88 carefully peeled off from the mold. The fluidic inlet and outlet were created with a biopsy punch.

89 Finally, the PDMS piece was treated with air plasma (Harrick) (Harrick Plasma, Ithaca, NY, USA)

90 for $1 \mathrm{~min}$. to promote adhesion and then pressure-bonded to a cover slip (\#1.5, $64^{\star} 50 \mathrm{~mm}$,

91 ClariTex) (Ted Pella, Inc., Redding, CA, USA) (Fig. 2D).

\section{Stem cell culture and retinal organoids initiation}

93 Retinal organoids were differentiated from genetically modified registered human embryonic

94 stem cells (hESCs) with green fluorescent protein (GFP) tagged to CRX gene which encodes

95 cone-rod homeobox protein and is specifically expressed in photoreceptor cells (WA01 line

96 expressing CRX-GFP) ${ }^{41}$. Stem cells were maintained by feeding mTeSR 1 media (STEMCELL

97 Technologies, Vancouver, BC, Canada) daily and passaged every 4-7 days by ReLeSR

98 (STEMCELL Technologies) when cells reached $\sim 80 \%$ confluency. Cells were expanded on

99 Vitronectin XF ${ }^{\mathrm{TM}}$ (STEMCELL Technologies) coated plates at $37{ }^{\circ} \mathrm{C}$ in a humidified $5 \% \mathrm{CO}_{2}$ 100 incubator (Nuaire, Plymouth, MN, USA). 
To initiate organoid formation, Accutase (Nacalai Inc, Kyoto, Japan) was added to the stem cells into a single cell suspension when 2 -dimensional culture reached $\sim 80 \%$ confluency. The 103 cells were then placed in a 800- $\mu$ m micro-well EZSPHERE 12-well plate (Nacalai) and centrifuged 104 at $100 \mathrm{~g}$ for $3 \mathrm{~min}$. to evenly distribute the stem cells throughout the bottom of each well. From day 1051 to 7 , the stem cells self-aggregated into embryonic bodies (EBs) in the EZSPHERE microwells. 106 From day 8, the EBs were seeded onto a 1\% growth factor reduced Matrigel $(r)$ coated culture 107 dish. The EBs spread onto the Matrigel and began 2D differentiation. Retinal eye fields were cut 108 from the Matrigel on day 50 and transferred to ultra-low attachment 24-well plates (Corning Costar) 109 (Corning, Corning, NY, USA) for 3D culture to be loaded into the bioreactor chip. Media used for 110 retinal organoid differentiation was modified from Zhong et al. ${ }^{42}$ From day 0 to 18 , the organoids 111 were gradually transitioned from mTeSR1 medium into neural induction media (NIM) containing 112 Dulbecco's modified eagle medium (DMEM)/F12 (1:1), 1\% N2 supplement (Gibco ${ }^{\mathrm{TM}}$ ) (Thermo 113 Fisher Scientific, Waltham, MA, USA), 1x minimum essential media non-essential amino acids 114 (NEAA) (STEMCELL Technologies), 1x L-glutamine (STEMCELL Technologies), and $2 \mu \mathrm{g} / \mathrm{ml}$ 115 heparin (Sigma-Aldrich, St. Louis, MO, USA), with daily media changes. From day 19 to 41 , the 116 media was switched to NIM containing DMEM/F12 (1:1) supplemented with $2 \%$ B27 supplement 117 (without vitamin A, Gibco), 1x NEAA, 1x L-glutamine, and 2mg/ml heparin. From day 42 and 118 beyond, the organoids were cultured with media containing DMEM/F12 (1:1) supplemented with $1192 \%$ B27 supplement (Gibco), 1x NEAA, 1x L-glutamine, 2ug/ml heparin, $100 \mu \mathrm{M}$ taurine (Sigma), 120 and $10 \%$ fetal bovine serum (FBS; Gibco). The media was changed 3 times a week and the 121 organoids were maintained at $37^{\circ} \mathrm{C}$ in a humidified $5 \% \mathrm{CO}_{2}$ incubator.

122 The organoids used in this study were all generated from the same batch. On day 87 of 123 differentiation, 15 RtOgs were randomly selected to load one each into every other chamber in 124 the bioreactor chip. After 37 days of on-chip culture, 3 RtOgs were selected for histology. The 125 remaining 12 RtOgs were divided into 3 groups of 4 RtOgs for qPCR gene expression analysis.

\section{Bioreactor system assembly and organoid loading}


The chip and the associated tubing were disinfected with $70 \%$ ethanol and $30 \mathrm{~min}$. in a UV 128 and ozone cool clave (CoolCLAVE Plus) (Genlantis, San Diego, CA, USA). Each chamber was 129 treated with anti-cell adherence solution twice (STEMCELL Technology) and washed by 130 Dulbecco's phosphate-buffered saline (DPBS) without calcium and magnesium (STEMCELL 131 Technology). The on-chip culturing system was assembled as shown in Fig. 2F. The media 132 reservoir was comprised of a $50 \mathrm{~mL}$ Steriflip-GP sterile centrifuge tube (MilliporeSigma, Burlington, 133 MA, USA) and a filter cap with a pore size of $0.22 \mu \mathrm{m}$.

134 Before loading the organoids, the chip chambers were sealed by pasting a slice of 135 MicroAmp $^{\mathrm{TM}}$ optical adhesive film (Thermo Fisher Scientific, Waltham, MA, USA) on the top 136 surface, then slowly withdrawing the syringe to apply negative pressure to fill the channel with 137 fresh media drawn from the media reservoir. Tubing clamps were then applied to block both the 138 inlet and outlet tubing, so that the adhesive film could be removed without disturbing the fresh 139 media level in the channel. One organoid was loaded into each chamber by $20 \mu \mathrm{L}$ pipette tips 140 with tip heads cut off. Lastly, the top of the chambers was resealed with sterile optical adhesive 141 film (Fig. 2E). The flow rate used for long-term culture was $250 \mu \mathrm{L} / \mathrm{h}$. Under this flow rate, $50 \mathrm{~mL}$ 142 media was sufficient for about 8 days of culture. When changing the media, the inlet and outlet 143 tubing were clamped and fresh media was refilled in the centrifuge tube. All these steps were 144 performed in an ESCO Class II Type A2 biosafety cabinet (Labculture, ESCO) (ESCO Micro Pte. 145 Ltd., Singapore) to avoid contamination. 


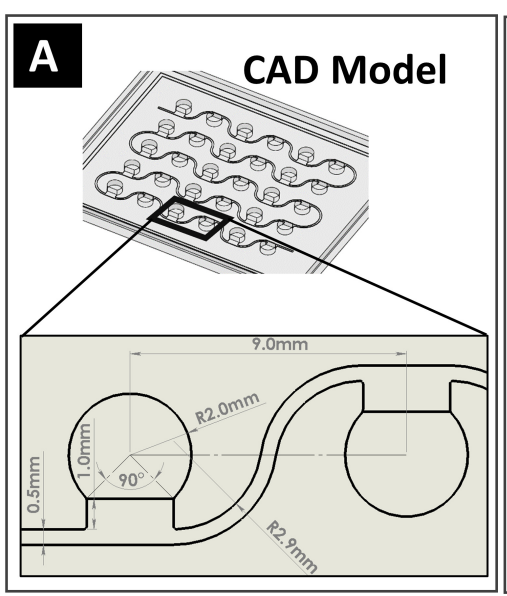

\section{B}

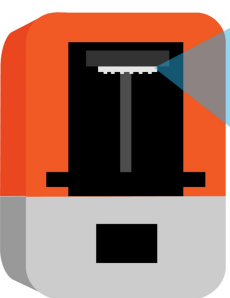

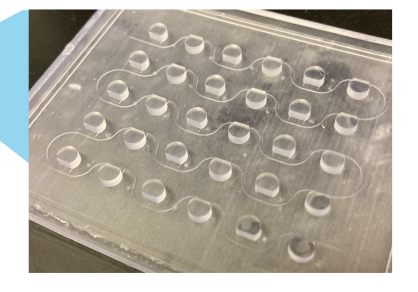

Mold

3D Printed Mold

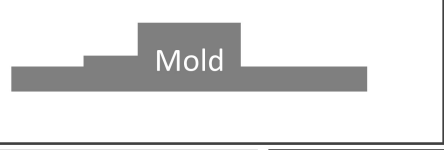

\section{C}

PDMS
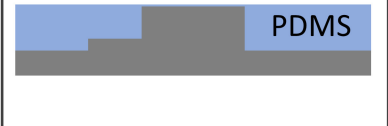

D Assembled Bioreactor

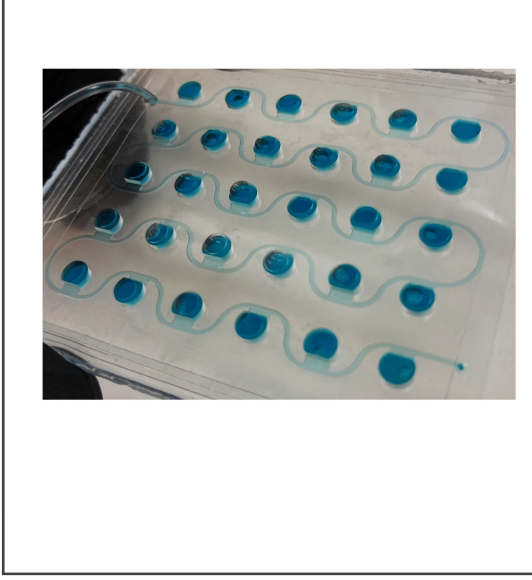

E

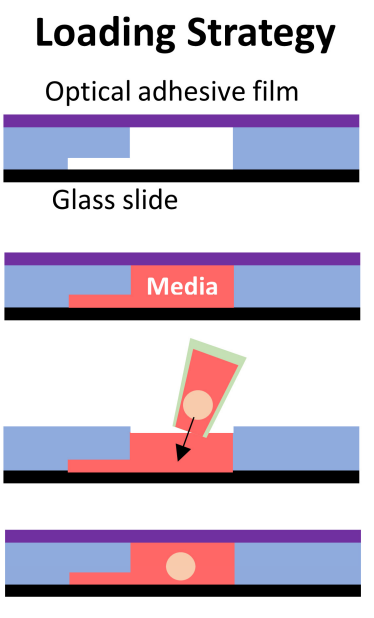

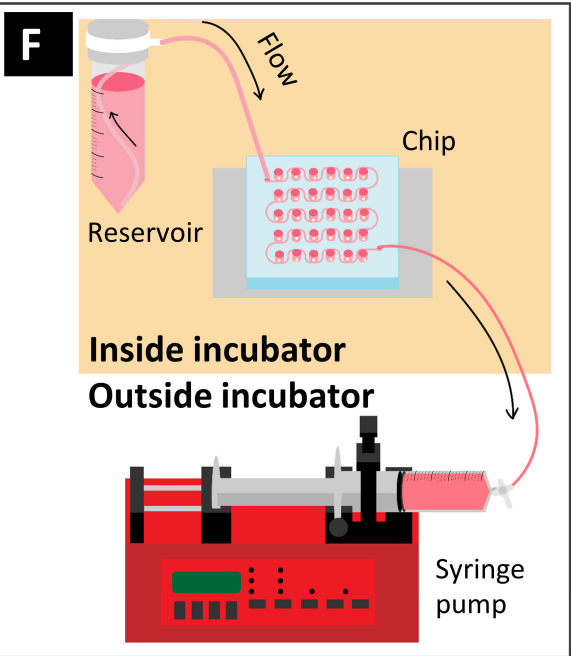

Figure 2: Fabrication methods. (A) Mold design with CAD software; (B) Mold printing; (C) PDMS casting on the mold; (D) Assembled bioreactor; (E) Cross section view of organoid loading procedure whereby microchannels were filled with media first, then an organoid was placed in the open well, and the wells were sealed using adhesive optical film; (F) On-chip culturing system assembly.

\section{In vitro dye test}

The dye test experiment was performed to compare the uniformity of the concentration in

154 chambers between the four different channel designs. Four chips with $3 \times 3$ chamber array were

155 fabricated with 2-mm chamber height. The channels were first filled with blue food dye solution

156 following similar steps as the organoid loading procedure (Fig. 2E). The flow was then blocked by

157 clamping both the inlet and outlet tubing, and the inlet was switched to a yellow dye solution. 
158 Lastly, a syringe pump was used to draw the yellow dye solution into the chip at a rate of 600 $159 \mu \mathrm{L} / \mathrm{h}$. The whole flow process was recorded with a camera. The grayscale value of each chamber 160 was obtained by ImageJ to quantify concentration changes of each chamber from the images.

$161 \quad$ Fluorescence life-time imaging

162 Fluorescence lifetime imaging (FLIM) was used to study the intrinsic fluorophore 163 Nicotinamide adenine dinucleotide (NADH) in the RtOg. The fluorophore's emission decay curve 164 was obtained by photon counting to calculate the fluorescent lifetime (Fig. 3A). FLIM data was 165 displayed on a phasor plot after Fourier transform, with the intensity decay curve of fluorescence 166 for each pixel represented by the $g$ and $s$ coordinates. Using this method, the decay and spectrum 167 for each pixel could be depicted on the phasor plot (Fig. 3B).

168 The metabolic trajectory was visualized with phasor approach ${ }^{43}$. The phasor plot has a 169 universal circle, with boundaries of each point representing a single exponential lifetime of one 170 type of molecule. Different components on the phasor plot followed a linear relationship, thus, the 171 ratio of the linear combination could be used to determine the fraction of each component. The 172 lifetime of free and lactate dehydrogenase-bound NADH was about 0.37 ns and 3.4 ns, 173 respectively ${ }^{44}$. Free $\mathrm{NADH}$ was linked to more glycolysis and a more proliferative state, while 174 bound NADH was correlated with more oxidative phosphorylation and a more differentiated state 17545. The lifetime of lipid was 7.89 ns associated with long lifetime species (LLS) (Fig. 3B), the 176 presence of which indicated oxidative stress ${ }^{46}$. The fraction of each component was calculated 177 as Fig. 3B suggested, F1/F2 was the free/bound NADH ratio, and F3 was the ratio of LLS. Based 178 on the above mechanism, we evaluated the metabolic state of RtOgs quantitatively by calculating 179 the free/bound NADH ratio and LLS ratio in representative image cross-sections. Qualitatively, 180 the metabolic differences were visualized by applying a pseudo color gradient to the phasor plot 181 (Fig. 3C). 
184 Tai multi-photon laser source (Spectra-Physics Mai Tai, Mountain View, CA). Imaging settings 185 used were as follow: 256 x 256 frame size, $1.66 \mu \mathrm{m}$ pixel size, $25.21 \mu$ s pixel dwell time and 8 bit 186 pixel depth. Emission laser passed through an MBS 690+ and an SBS SP 610 filters and the 187 lifetime data was collected by the photomultiplier tube (H7422p-40, Hamamatsu, Japan) and a320 188 FastFLIM FLIMbox (ISS, Champaign, IL). Before imaging, the system was calibrated on 189 frequency factor and lifetime by coumarin 6 solution with the known lifetime of $2.5 \mathrm{~ns}$. FLIM data 190 were collected after 100 counts in the brightest pixel of the image were acquired.

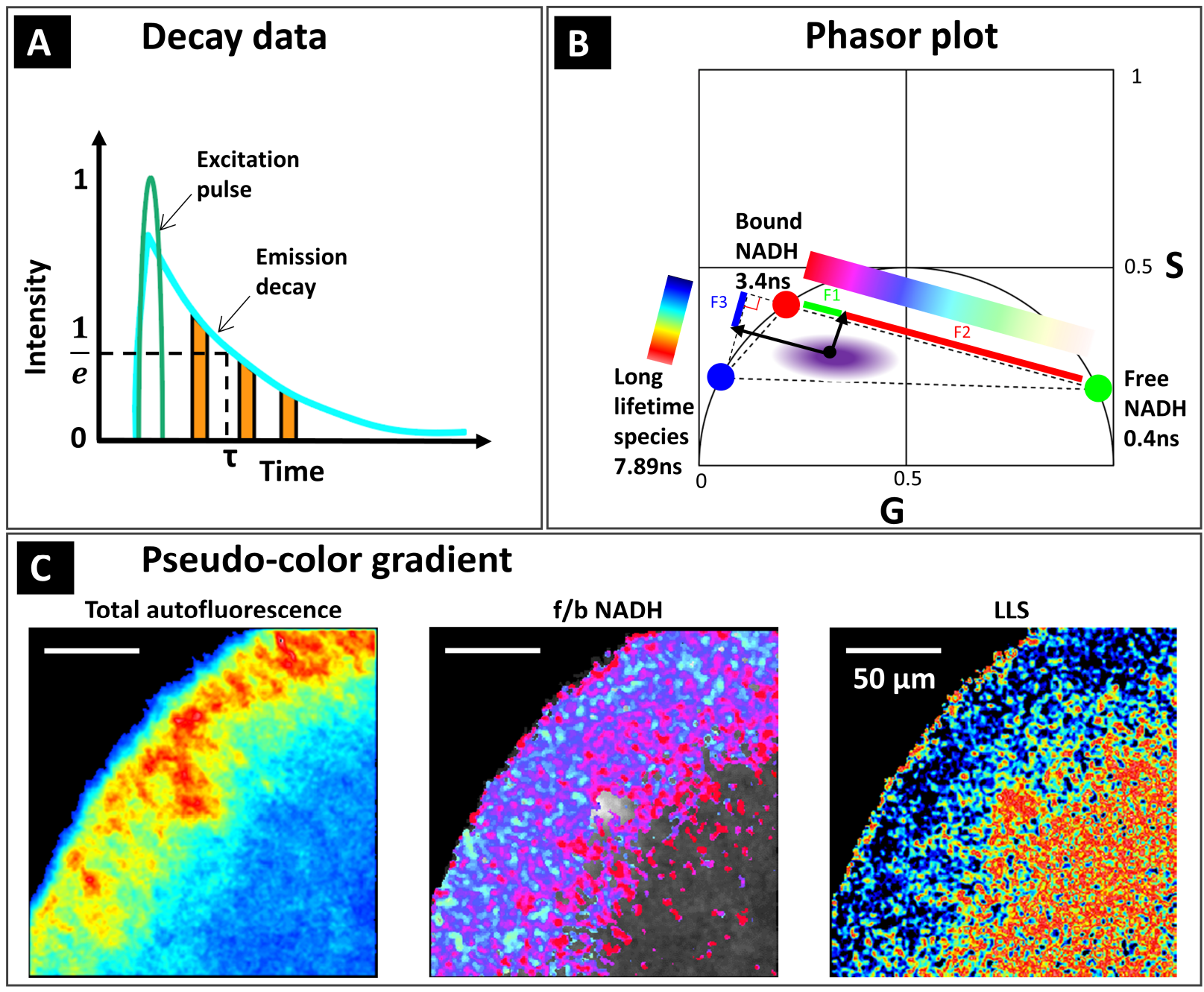


Figure 3: Fluorescence lifetime imaging and analysis using the phasor approach. (A)

193 Fluorescence lifetime was acquired by quantifying emitted fluorescent photon over time after an

194 excitation pulse was supplied to obtain an emission decay curve; (B) Phasor plot produced a 2-

195 dimensional space for intrinsic fluorophors with different lifetimes corresponding with different

196 types of metabolism (oxidative phosphorylation favors bound NADH and glycolysis favors free

$197 \mathrm{NADH}$ ) and different amounts of oxidative sress (long lifetime species). The free/bound NADH

198 ratio and long LLS ratio were obtained by calculating projecting the 3 dimensional photon count

199 histogram onto the Bound-Free axis and LLS axis respectively; (C) A representative images of

200 RtOg analyzed by the phasor approach. The autofluorescence images encapsulated all total

201 fluorescenee, while the $\mathrm{f} / \mathrm{b}$ NADH and LLS are pseudocolor images based on the phasor analysis

202 of quantized fluorescent emission. f/b NADH was free to bound NADH ratio. LLS was long lifetime 203 species.

\section{Phase contrast imaging}

205 The phase contrast microscopy images were acquired using an Olympus IX71 (Olympus, 206 Tokyo, Japan) and a QICAM FAST1394 CCD camera (Teledyne QImaging, Surrey, BC, Canada) 207 under two magnifications by UPlanFL N 4x/0.13 PhL and UPlanFI 10x/0.30 PhL objectives.

\section{Green fluorescent protein imaging}

209 Green fluorescent protein images were acquired using a Zeiss LSM 780 microscope using 210 Plan-Apochromat 20x/0.8 M27 objective (Carl Zeiss, Jena, Germany). The excitation wavelength 211 was $488 \mathrm{~nm}$ with a pixel dwell time of $1.58 \mu \mathrm{s}$. We used the frame size of $512 \times 512$ pixels and 212 each pixel is $0.42 \mu \mathrm{m}$.

\section{Quantitative polymerase chain reaction analysis}

214 The primers for qPCR test were listed in Table S1 (Qiagen, Germantown, MD, USA). We 215 used 12 retinal progenitor and photoreceptor genes and 1 housekeeping gene to identify and 
216 quantify the gene expression profile in retinal organoids. Human adult retinal tissue was used as

217 a positive control $(n=3)$. Each RtOg sample was analyzed at days 122 to 124 of differentiation

218 ( $n=3$ for both static and chip groups). Each sample consisted of 4 individual RtOgs. Trizol reagent

219 (Qiagen), DNase I digestion (Fisher, Waltham, MA, USA), and phenol-chloroform extraction

220 (Fisher) were used to isolate RNA, and an $\mathrm{RT}^{2}$ cDNA synthesis kit (Qiagen) was used to

221 synthesize cDNA. RT2 SYBR Green with ROX qPCR master mix (Qiagen) was used for

222 amplification, which was performed under the following conditions: $95^{\circ} \mathrm{C}$ (15 minutes), 40 cycles

223 at $95^{\circ} \mathrm{C}(15$ seconds each $), 55^{\circ} \mathrm{C}$ (30 seconds each) and $72^{\circ} \mathrm{C}$ (30 seconds each). The annealing

224 temperature was $60^{\circ} \mathrm{C}$. The double delta cycle threshold $(\mathrm{Ct})$ method was used to calculate the

225 fold expression, and day 0 undifferentiated hESC (line CSC14) was used as a control. For the

226 purpose of analysis and heatmap generation, non-detected amplification in the control tissue and

227 organoids were assigned cycle threshold values of 40 . Heat maps were generated using

228 Graphpad Prism software (Graphpad Software LLC, La Jolla, CA, USA), the heat map has the

229 value of $\log _{2}$ (Fold Expression), which is equal to $-\Delta \Delta \mathrm{C}_{\mathrm{t}}$.

\section{$230 \quad$ Histology and hematoxylin-eosin staining}

231 RtOgs at day 124 of differentiation were fixed with cold $4 \%$ paraformaldehyde in $0.1 \mathrm{M} \mathrm{Na-}$

232 phosphate buffer for 1 hour (static: $n=2$; bioreactor: $n=3)$, cryoprotected (30\% sucrose) and

233 frozen in optimal cutting temperature compound. Cryostat sections $(10 \mu \mathrm{m})$ were stored at $-20^{\circ} \mathrm{C}$.

234 Slides in both groups were stained using hematoxylin and eosin and then imaged on an Olympus

235 BXH10 (Center Valley, PA, USA) using an Infinity 3-1U camera (Lumenera, Ottawa, Ontario, 236 Canada).

\section{$237 \quad$ Statistical analysis}

238 Data in the plot were presented as means with standard deviations. Graphpad Prism software 239 was used for all statistical analyses. In the GFP MFI, free/bound NADH and LLS ratio figures, 
one-way ANOVA test was performed. In the qPCR heatmap, two-way ANOVA test was performed.

241 The significance was determined by a $p$ value less than 0.05 .

\section{RESULTS}

\section{$244 \quad$ Microfluidics design and testing}

245 The chip design was a miniature version of a conventional tissue culture plate with the 246 distance between chambers matching a 96-well plate to retain microscope compatibility.

247 Preliminary designs in which chambers were located on one side of a $1000 \mu \mathrm{m}$ wide perfusion

248 channel revealed two problems: 1) uneven concentration among chambers and 2) low mass

249 transfer efficiency (Fig. S1). Therefore, not all wells in the preliminary design received comparable

250 media perfusion. To optimize the design, three different variables that affected the mass transfer

251 rate were evaluated with COMSOL simulation: channel width, channel alignment and chamber 252 height.

253 The channel width determined the cross-section area and thus affected the flow velocity $(v)$ 254 as indicated in Equation (1). Holding volume flow rate $(Q)$ constant, the larger the cross-sectional 255 area $(A)$, the slower the flow velocity $(v)$ would be.

$$
Q=A v
$$

257 According to the definition of Péclet number $\left(P e_{L}\right.$, the ratio of advective transport rate to 258 diffusive transport rate, Equation (2)), larger flow rate would lead to higher advective transport 259 rate, accelerating mass transports.

$$
P e_{L}=\frac{L v}{D}
$$

261 where $L$ is the characteristic length, $v$ the local flow velocity, and $D$ the mass diffusion 262 coefficient. Therefore, narrowing the channel width would facilitate an increase in flow velocity. 263 Based on the simulation results, under both flow rates, the narrow channel $(500 \mu \mathrm{m})$ designs 
264 showed faster mass transfer (Figs. 4A-B) and therefore, a theoretically faster delivery of media to 265 each culture chamber.

266 Incompressible fluid flow within the microfluidic device, due to its small size, should be 267 laminar with a parabolic velocity profile when fully developed. As a result, the velocity next to the 268 channel walls should be close to zero. Thus, the designs with all chambers on one side of each 269 channel should show a higher velocity in the fluid close to the channel wall connected to a 270 chamber and that on the opposite wall. Figs. 4A-B show that single-sided channel showed a 271 concentration gradient from the first chamber to the last chamber in each row and then entire 272 series of chambers. This difference was even more pronounced in larger series of $5 \times 6$ chambers 273 (SFig. 1). To minimize this effect, a serpentine channel was designed to promote comparable 274 media diffusion from both sides of the channel. To further improve concentration distribution, a 275 mixer unit was added between each chamber ${ }^{47}$. Empirical evidence with a larger $5 \times 6$ array of 276 organoids demonstrate significant variability between chambers in each row (SFig. 1). Simulation 277 demonstrated that narrow channels with or without mixer showed comparable qualitative 278 performance as indicated with the color map representation of the concentration variations 279 between the first and last culture chambers in each row and those between rows.

280 The third variable evaluated with simulation was the chamber height. By tracking the point 281 concentration on the same top corner of each chamber, the 3D COMSOL simulation results 282 showed that doubling the height of the chamber to $4 \mathrm{~mm}$ caused a dramatic change $(4 \sim 10$ folds 283 difference) in mass transport efficiency (Fig. 4D). To maximize the transport efficiency, we chose $2842 \mathrm{~mm}$ as our final chamber height for bioreactor fabrication. Fig. 4E shows the 3D concentration 285 patterns in four different bioreactor designs. 


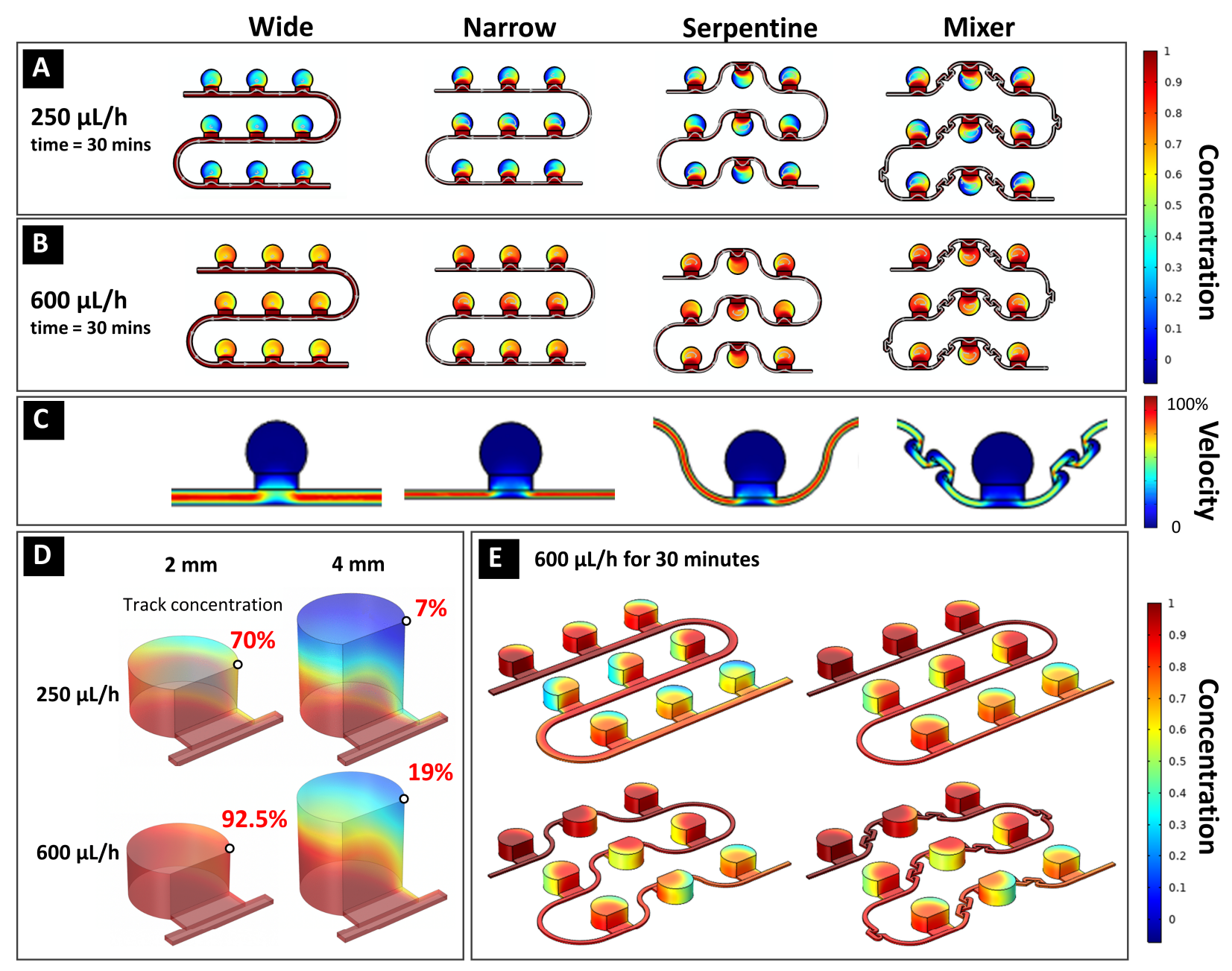

287 Figure 4: COMSOL simulation of 4 different channel designs. (A) Concentration distribution

288 after 30 minutes of slow flow $(250 \mu \mathrm{L} / \mathrm{h})$; (B) Concentration distribution after 30 minutes of fast

289 flow $(600 \mu \mathrm{L} / \mathrm{h}) ;(\mathrm{C})$ Velocity distribution - zero velocity in all chamber demonstrated shear stress-

290 free culture environment. A single culture chamber and adjacent flow channels is shown because

291 focal flow velocity was identical for every culture chamber and interconnecting microfluidic

292 channels in the linear series; (D) Mass transfer efficiency comparison between different height

293 chambers under two different flow rates after 30 minutes. Black circles represent location of

294 concentration determination at 30 minutes; (E) 3D concentration pattern of four different designs. 
296 To confirm simulation results and examine the functionalities of the four designs, a dye test 297 was performed to confirm the optimum design for culturing RtOgs. A $3 \times 3$ chamber array was 298 fabricated for each channel design with a $2 \mathrm{~mm}$ tall culture chamber. Blue dye was used to fill 299 each channel followed by 30 and 48 minutes of $600 \mu \mathrm{L} / \mathrm{h}$ flow of yellow dye (Fig. 5A). The 300 grayscale photogrammetry from pictures taken on each chamber were quantified (Fig. 5B). The 301 serpentine channel with mixer design showed the smallest standard deviation, indicating that this 302 design had the most uniform concentration among the four. The serpentine channel without mixer 303 exhibited the next best performance based on variability after 48 minutes of flow. The simple 304 serpentine channel without mixer showed higher fabrication success with 3D printing and lower 305 probability of trapped air bubbles in the microfluidic channels than the serpentine channels with 306 mixer. 


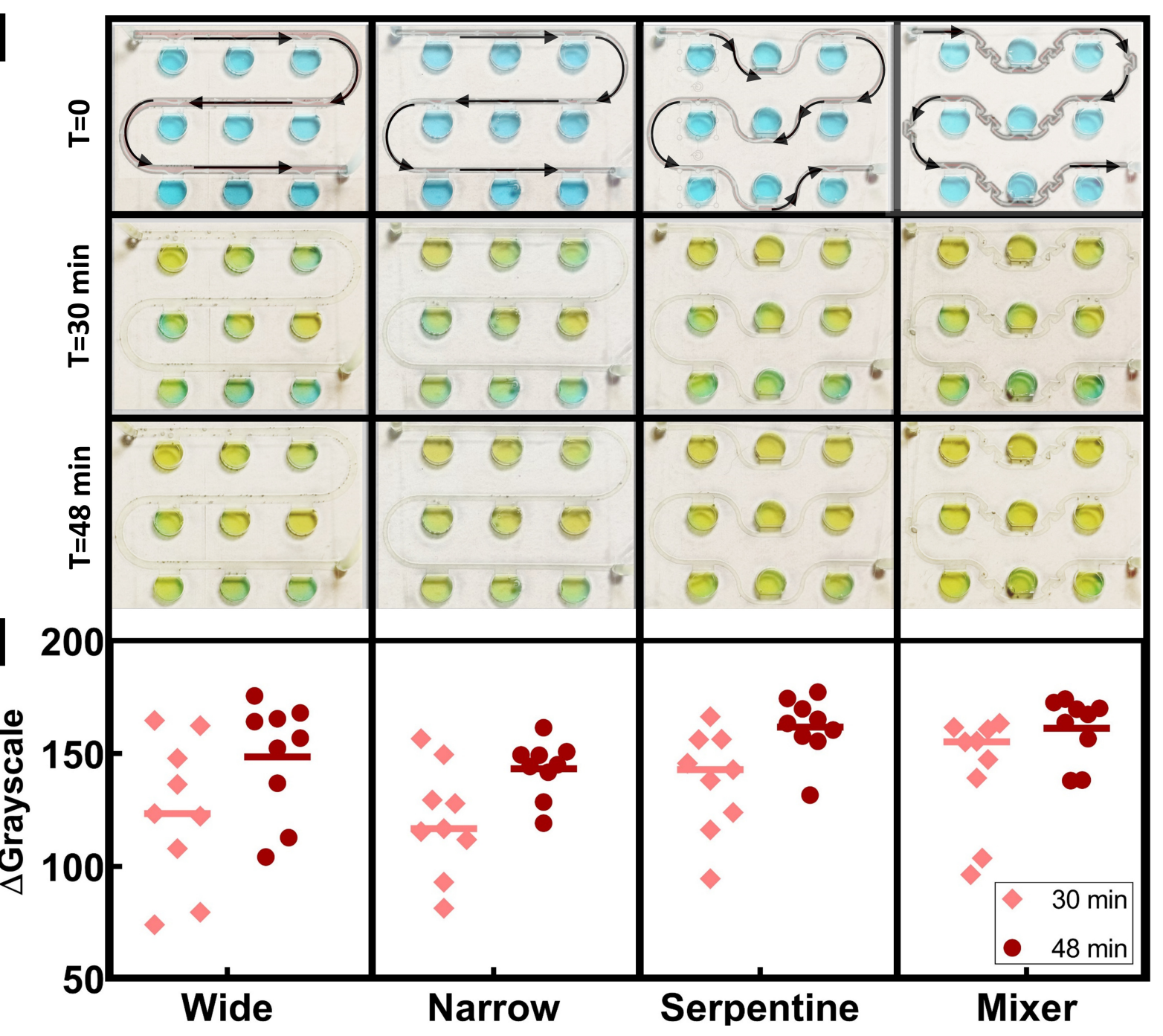

Figure 5: Flow and concentration distribution experiment with dye. (A) Diffusion pattern of

four different designs (flow rate was $600 \mu \mathrm{L} / \mathrm{h}$ ); (B) Grayscale change of each well after 30 minutes

310 and 48 minutes.

\section{Retinal organoid culture methods comparison}

\section{$312 \quad$ Phase contrast imaging}

313 The RtOgs used in this study were differentiated from human embryonic stem cells.

314 Representative phase contrast images in Figs. 6A-E showed the key stages of RtOg

315 differentiation. The EZSPHERE microwell was used to form stem cells into uniformly sized

316 embryonic bodies. Eye fields cut from Matrigel were maintained in ultra-low attachment 24-well 
317 plate as they assembled into RtOgs. In this study, RtOgs were put on the bioreactor on day 87 of 318 differentiation.

319 Figs. 6F-M showed two representative RtOgs in both groups on day 88 and day 124 of 320 differentiation, respectively. The observable transparent and laminar outer surface, which was

321 observed in both groups, indicated development of a photoreceptor layer. For the bioreactor 322 culture method, the RtOgs tended to grow beside the chamber wall (Fig. 6I). The bioreactor was

323 treated with anti-cell adherence solution twice (STEMCELL Technology) before introducing

324 RtOgs, which effectively prevented adhesion during the first three weeks. However, after three

325 weeks, some of the RtOgs began attaching to the chamber walls without extending along the

326 PDMS surface. As a result, the RtOgs continued to undergo 3D differentiation albeit displaying

327 slight deformation due to the physical contact with the chamber wall.

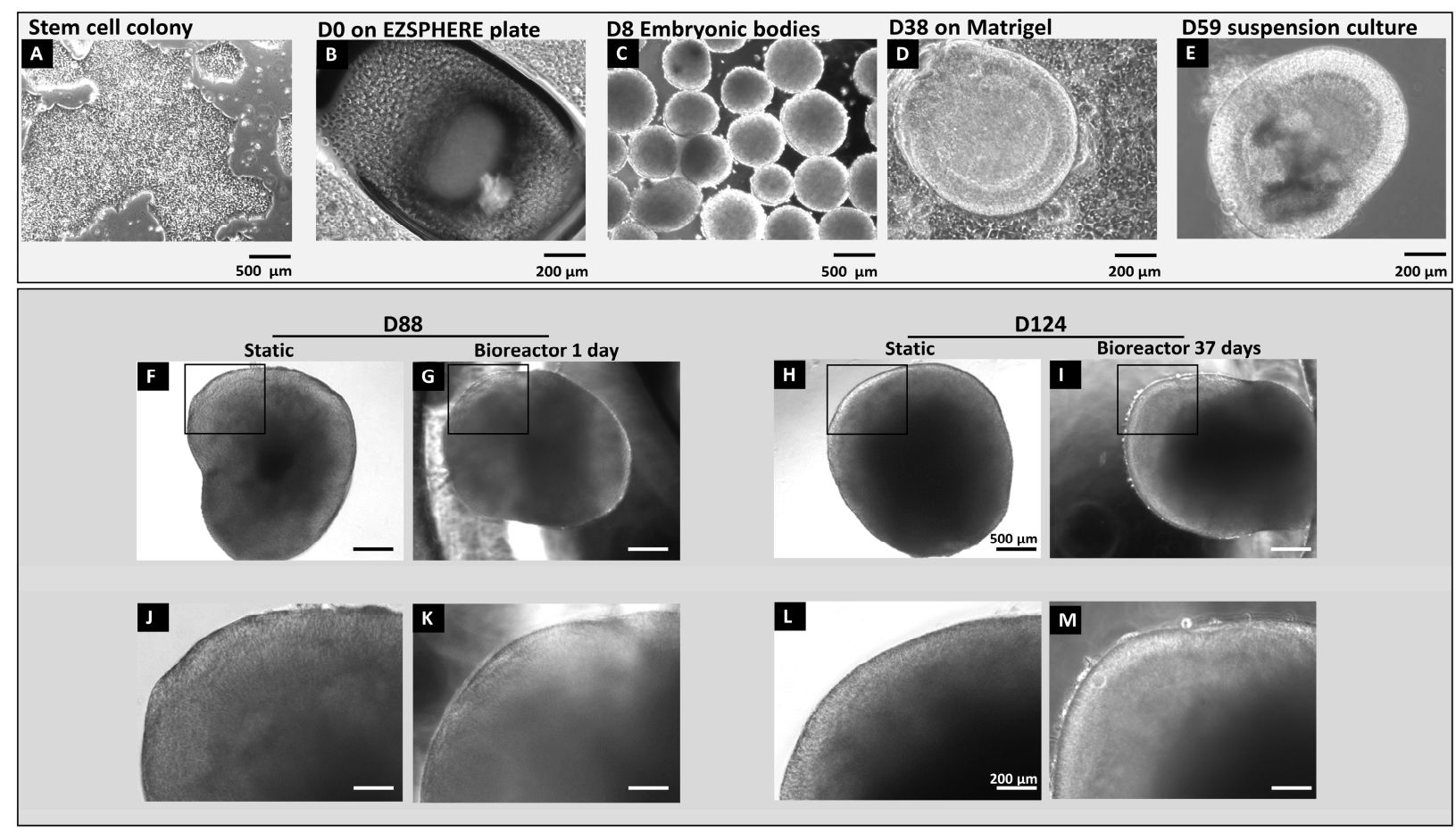

329 Figure 6: Phase contrast images. (A) Human embryonic stem cell colony; (B) Day 0 of

330 differentiation, dissociated stem cells in EZSPHERE microwell plate (well size: $800 \mu m$ ); (C) Day

3318 of differentiation, embryonic bodies ready for seeding on Matrigel; (D) Day 38 differentiation on

332 Matrigel; (E) Retinal organoid in suspension culture on day 59; (F-G) RtOgs on day 88 of 
333 differentiation; ( $\mathrm{H}-\mathrm{I})$ RtOgs on day 124 of differentiation; (J-M) Higher magnification images

334 correspondeng to images above in F-I.

\section{$335 \quad$ Fluorescence lifetime imaging}

336 The bioreactor chip platform was supplied with nutrients continuously while the RtOgs in 337 conventional dish culture received nutrient exchange every 3 days. This distinction might cause

338 metabolic differences in the long-term. The hypothesis is that chip cultured RtOgs would 339 experience less oxidative stress caused by reactive oxygen species (ROS), and the sufficient 340 nutrients supply would benefit RtOgs survival and maturation. We previously used FLIM for live 341 RtOg characterization ${ }^{48}$. FLIM was used to measure the metabolic activity in a non-invasive and 342 non-destructive way as described in the method section.

343 Four imaging modalities were used to visualize the same cross-section in RtOgs.

344 Conventional fluorescence microscopy demonstrated greenfluorescnt protein in photoreceptors 345 and their progenitors (Figs. 7E-H). Multiphtoton infrared stimulation was used to acquire total 346 autofluorescent images showing the total NADH (Figs. 7A-D), which delineated cellular structures 347 and viability of RtOgs. Multiphoton lifetime imaging revealed metabolic changes in NADH from its 348 free to bound form and their associated free:bound ratio (f/b NADH) (Figs. 7I-L). Long lifetime 349 species analysis highlighted oxidative stresses in the tissues (Figs. 7M-P).

350 From day 98 to day 120 of differentiation, RtOgs in both groups shifted from more glycolytic 351 (yellow-green) to more oxidative phosphorylation (red) (Figs. 7I-L), the difference was significant 352 (Fig. 7S), which suggested that RtOgs on day 120 were more differentiated from a stem cell state 353 (glycolytic) 49, 50. Furthermore, greater metabolic signature from NADH was present from 354 bioreactor cultured organoids (Comparing Figs. 7K and 7L). When compared within groups, the 355 bioreactor cultured RtOgs had no significant different f/b NADH ratio on day 120 (Fig. 7S), which 356 indicated that RtOgs in both groups obtained comparable differentiation states. The LLS images 357 showed a distinct color difference between two groups (Figs. 7M-P). RtOgs on the bioreactor had 358 experienced significantly lower oxidative stress than RtOgs in static culture at both imaged 
timepoints, and both groups increased LLS ratio across days (Fig. 7T). The time-dependent 360 metabolic shifts and the metabolic difference between two groups were also reflected on G-S 361 phasor plot, which provided the metbolic fingerprint of each group of samples. The measured 362 RtOgs were located at four different clusters (Fig. 7R).
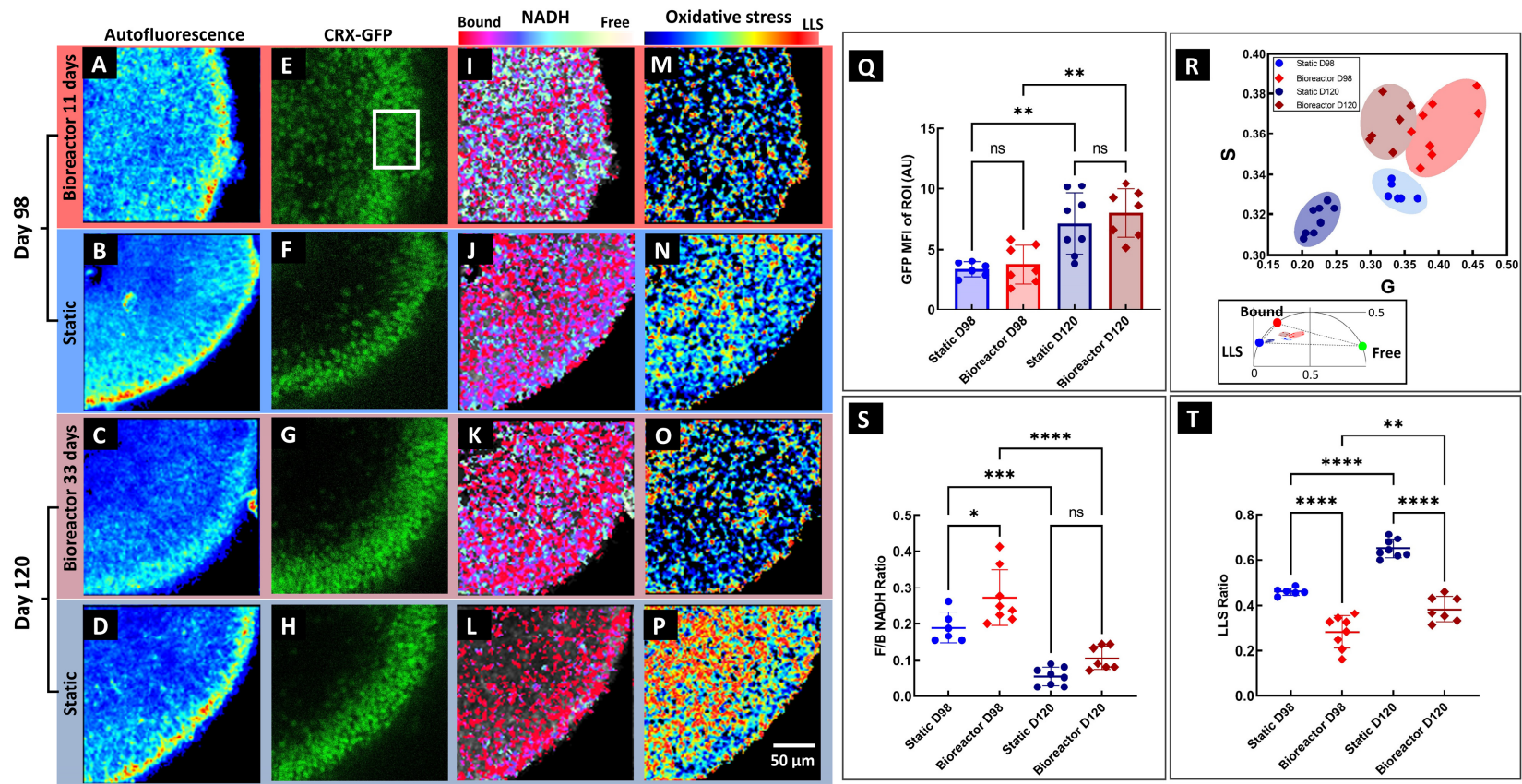

Figure 7: Qualitative and quantitative comparison of RtOgs in two culture methods. (A-D)

Total NADH autofluorescence images showed the cellular structures within the RtOgs' cross

section; (E-H) Fluorescence images showed distinct cell nuclear layer corresponding to the CRX-

367 GFP fusion protein localized in nuclei; (I-L) Pseudo color-coded free/bound NADH distribution

368 and (M-P) LLS distribution images were generated based on photon lifetime locations within the

369 2-dminensional phasor space; (Q) The mean fluorescence intensity of GFP signals at region of

370 interest (white box in Fig. 7E) (One-way ANOVA test was performed: Static D98, $n=6$; Bioreactor

371 D98, $n=7$; Static D120 n = 8; Bioreactor D120, $n=7)$; (R) Scatter plot of imaged RtOgs and their

372 location on the FLIM phasor diagram; (S) Plot of free/bound NADH ratio to evaluate metabolism

373 (higher $\mathrm{f} / \mathrm{b}$ value represented glycolysis, and lower $\mathrm{f} / \mathrm{b}$ indicated greater oxidative phosphorylation;

374 (T) Plot of LLS ratio to evaluate oxidative stress. (One-way ANOVA test was performed: Static 375 D98, n = 6; Bioreactor D98, n = 8; Static D120 n = 8; Bioreactor D120, $n=7$ ). 
Gene expression profile

377 We also examined the RtOgs' gene expression by fluorescence imaging and qPCR analysis. 378 RtOgs in both groups displayed a thick nuclear outer layer which expressed CRX gene on day 379120 of differentiation (Figs. 7E-H). The mean fluorescent intensity (MFI) of the selected outer 380 surface region showed no significant difference between static and bioreactor cultured RtOgs on 381 day 98 and 120. However, both groups had a significant increase of MFI over time, which 382 suggests an increase of CRX expression during RtOgs differentiation (Fig. 7Q).

383 The qPCR test provided a comprehensive retina related gene profile. Fig. 8A showed that

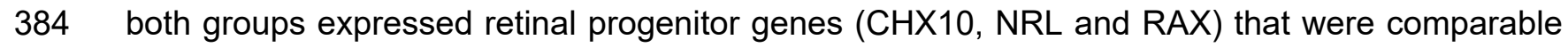
385 to those of human adult retina. Both groups also expressed rod and cone genes including RCVRN, 386 ARR3, SAG, PRPH2, GNAT and GNAT2. However, both groups had very little mature 387 photoreceptor gene expression, which was expected, as RtOgs typically do not reach full maturity 388 until day 150 200 of differentiation. The gene expression level showed no significant difference 389 between static group and RtOgs group (two-way ANOVA test, $p>0.05$ ). However, RtOgs on the 390 bioreactor expressed more OPN1 MW than static group ( $p=0.0633)$, which implied that micro391 millifluidic bioreactor might be the optimal platform for photoreceptor maturation.

$392 \quad$ Hematoxylin-eosin staining

393 Lastly, the RtOgs maintained in both conventional static culture or the bioreactor were fixed 394 on day 124 of differentiation and frozen sectioning performed to acquire hematoxylin and eosin 395 (H\&E) cross-sections. Representative images are shown in Figs. 8B-E. RtOgs in both groups 396 demonstrated clear and compact outer nuclear layer on the organoids surface, which suggested 397 the formation of photoreceptor layer. RtOgs maintained in conventional culture demonstrated lacy 398 vacuolization of the inner tissues, whereas the same was not as prominent in the bioreactor399 maintained organoids. 

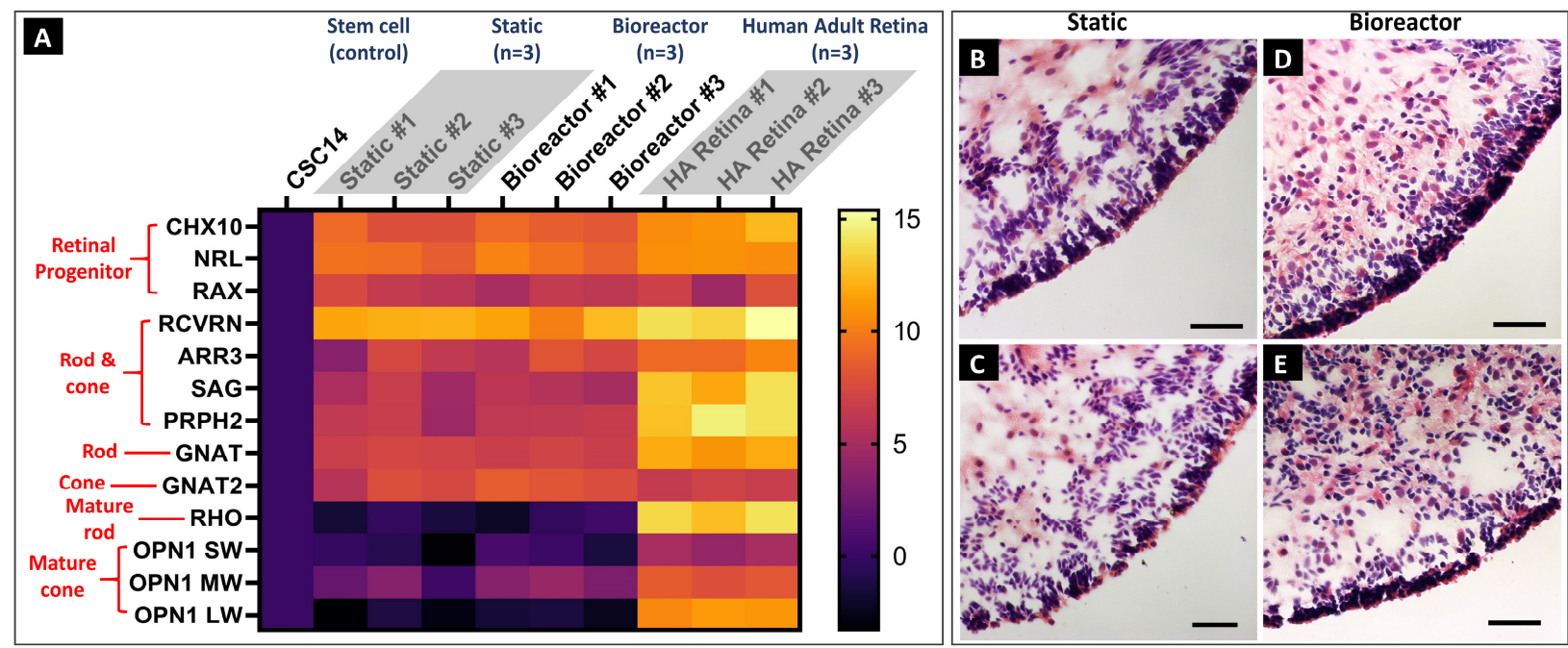

Figure 8: Gene profile and representative histology images of different experimental

groups. (A) The CSC14 stem cell was used as negative control and calibrator for all other groups.

Each sample in each experimental group contained 4 RtOgs. The human adult $(\mathrm{HA})$ retina

samples were set as positive controls. (B) Four Representative RtOgs in static culture (B-C) and bioreactor culture (D-E) on day 124 of differentiation (scale bar: $50 \mu \mathrm{m}$ ).

\section{DISCUSSION}

The overall goal of this study was to improve current RtOgs culture techniques by reducing manual labor required for organoid culture and improve the RtOgs reproducibility and quality. Two main differences between in vivo retina and conventional in vitro RtOg culture: consistency of

411 nutrition supply and fluid mechanical stability. In the human body, the visual system is the highest energy-consuming system in the brain ${ }^{51}$ and photoreceptor cells are identified as the most metabolically active cell. Retinas in the human body are continuously nourished by the dual blood

414 supply from the choriocapillaris and the retinal vasculature. Additionally, the retina in vivo is 415 isolated from mechanical forces, by virtue of the non-compressible fluid contents for the globe 416 and the outermost structural support provided by the sclera. In its natural configuration, the 417 photoreceptors are isolated from dynamic fluid forces, whereas organoids in tissue culture 
418 develop photoreceptors surrounded by turbulence in multi-well culture plates. Shear stress can 419 impact the stem cell differentiation phenotypes ${ }^{52}$, destroy circulating tumor cells ${ }^{53}$, induce cells' 420 metabolite production ${ }^{54}$, and cause RtOgs to lose the outer segment like structures ${ }^{55}$. Loss of 421 photoreceptor outer segments is also seen clinically in patients with retinal detachments who 422 develop glaucoma as a consequence of outer segment shedding ${ }^{56}$.

423 Bioreactors for organoid culture to remedy the drawbacks of conventional tissue culture have 424 been described in recent years. Existing challenges for bioreactor designs include minimizing the 425 volume of media used, minimizing shear stresses on tissues and reducing their incompatibility 426 with longitudinal non-invasive imaging. At the macro level, stirred and rotating wall vessel (RWV) 427 platforms have been used for retinal organoid differentiation. The former has been shown to 428 produce retinal organoids with improved laminar stratification and increased yield of 429 photoreceptor cells with outer segment structure, with drawbacks of damage to these fragile 430 structures from sheer stress ${ }^{55}$. The latter has the advantages of improved differentiation, easy 431 use, and high nutrient transfer, and has also been used to culture bladder, lung, intestinal, and 432 vaginal epithelial cell types into three-dimensional cell aggregates ${ }^{31},{ }^{57-60}$. However, these larger 433 systems share the disadvantage of high cost due to the high volume of media required to maintain 434 the organoids ${ }^{34}$. At an intermediate scale is the millifluidic system used to manipulate fluids for 435 organoid maintenance ${ }^{36}$. These bioreactors have been used for development of kidney organoids 436 and long-term maintenance of human midbrain and liver organoids $29,61,62$. Millifluidic systems 437 have the advantages of supporting relatively high flow rates, cell-cell interaction, and less frequent 438 media changes and thus less organoid perturbation but has the disadvantage of intermediately 439 high volume and cost, and low throughput. At the microscale, microfluidic devices have the added 440 advantages of lower volume and lower cost compare to millifluidics ${ }^{36}$. Microfluidic devices have 441 been used to culture human intestinal, lung, hepatocyte, and cardiac organoids, ${ }^{63-65}$. For both the 442 milli- and microfluidic devices, shear stress can be minimized by placing organoids in wells or 
443 chambers at a set distance from the flow channels. Therefore, we sought to reduce shear stress

444 while creating a perfused environment to house and isolate individual organoids for long term 445 non-invasive imaging.

$446 \quad$ First, we developed a hybrid bioreactor design that incorporated both micro and milifluidic 447 components. This design was made possible from the novel fabrication method relying on SLA 448 3D printers to create a mold incorporating micro-, milli- and even macroscopic features (Figs. 2A449 D). Thanks to the development of additive manufacturing techniques, we were able to utilize the 450 SLA 3D printing to combine the micro and milli features easily in one single design. 3D printing 451 also enabled rapid prototyping bioreactor designs to iteratively optimize the design. Cost savings 452 and facility compared with traditional microfabrication methods offers additive manufacturing as 453 a useful approach to manufacturing bioreactors ${ }^{66}$.

454 We used computer simulation to first demonstrate that each millifluidic culture chamber could 455 be supplied with media from a microfluidic channel. We evaluated flow velocity inside culture 456 chambers and found no active flow (Fig. 4C), which satisfied the design goals to minimize 457 turbulence and shear stresses on retinal organoids by eliminating fluidic movement in the culture 458 chamber. We further optimized channel geometry relative to the culture chambers, flow rate of 459 media through the bioreactor and inclusion of microfluidic mixers between each culture chamber.

460 The endpoint for determining success in each design iteration was comparing uniformity of media 461 composition in each culture chamber. We performed both COMSOL simulations in silico (Fig. 4) 462 as well as dye tests in vitro (Fig. 5). In silico simulations demonstrated that narrow microfluidic 463 channels $(500 \mu \mathrm{m}$ wide $\times 200 \mu \mathrm{m}$ tall) allowed greater mass transfer than wider microfluidic 464 channels $(1 \mathrm{~mm}$ wide $\times 200 \mu \mathrm{m}$ tall). We also observed in silico that high flow rate (250 vs 600 $465 \mu \mathrm{L} / \mathrm{hr}$ ) also improved mass transfer into culture chambers (Fig. 4B). In vitro dye tests to confirm 466 in silico modeling predictably revealed that bioreactor designs with all culture chambers arranged 467 on the same side of the microfluidic channel suffered from diffusion from a single side of the 
channels laminar flow. This resulted in the first chamber in each row of the microfluidic series to 469 have the highest mass transfer of fresh media, while the last chamber had the lowest (Fig. 5A). 470 To overcome this limitation, we designed a bioreactor with serpentine microfluidic flow line and 471 culture chambers on alternating sides of the microfluidic flow line. These designs were simulated 472 in silico to reveal improved concentration uniformity in each culture chamber compared with 473 straight channel designs. In vitro dye testing confirmed that media concentration variability 474 between all wells was improved by the serpentine design (Fig. 5B). Finally, we introduced mixers 475 in the flow channel to determine if mixing would improve culture chamber concentration uniformity. 476 In silico simulation demonstrated improved chamber concentration uniformity over the serpentine 477 channel design (Fig. 5A). In vitro dye testing demonstrated a marginal improvement when the 478 mixer was included than when it was not. A decision based on practical implementation was made 479 to exclude the mixer because of the higher probability of trapping bubbles in the mixer elements 480 as well as the mixer having tapered features that exceeded the resolution of the 3D printers 481 employed.

A second major requirement for our design was to enable long-term imaging of retinal 483 organoids maintained in perfused culture. The bioreactor chip design included glass cover slips 484 to seal the microfluidic circuit. Glass cover slips are thinner than microscope slides and, therefore, 485 suitable for both multiphoton imaging and conventional fluorescence microscopy. Multiphoton 486 imaging relies upon optimally efficient photon capture, and thicker glass slide reduces captured 487 photons below threshold of practical imaging.

$488 \quad$ Archberger et al. demonstrated a chip platform containing tissue chambers seeded with RPE 489 and fed with media via a porous membrane to mimic vasculature in the retina ${ }^{67}$. This platform 490 emphasized human physiological fidelity and minimized shear stress. However, a limitation was 491 the relatively short 7-day maintenance of the chip platform for organoid culture. After optimizing 492 our bioreactor design, we evaluated its performance in sustaining retinal organoids. The protocol 
493 for loading organoids into the bioreactor was determined as described in Figs. 2E-F. In this body 494 of work we sought to evaluate the bioreactor's ability to maintain RtOgs for 1 month. We 495 compared organoids differentiated to 88 days in vitro that were either placed in the bioreactor for 49637 days or remained in conventional plate culture. Non-invasive functional imaging of metabolism 497 and oxidative stress, sustained development of photoreceptors on the organoids outer layer, and 498 terminal PCR analysis of RtOg tissue were endpoints for comparing culture conditions. Phase 499 contrast microscopy revealed that RtOgs maintained in conventional culture and bioreactors 500 developed a comparable semi-translucent outer layer. Qualitatively, we observed slightly more 501 photoreceptor outer segments in the RtOgs maintained in the bioreactor. The fluorescent reporter 502 GFP linked to nuclear CRX served as a marker for photoreceptors and their progenitors. We 503 observed an insignificant difference in the fluorescence intensity of $\mathrm{GFP}^{+}$nuclei in organoids of 504 the same age when they were cultured in the bioreactor or conventional culture (Fig. 7Q), 505 suggesting the cellular composition is likely similar between RtOgs maintained in either culture 506 condition.

$507 \quad$ However, we observed a statistically significant difference in metabolism and oxidative stress 508 between organoids maintained in the bioreactor versus conventional culture (Fig. 7). Organoids 509 in the bioreactor had significantly higher total NADH signal. It is worth noting that bioreactor 510 cultured RtOgs at both timepoints presented similar f/b NADH ratio as those in static culture, 511 indicating similar differentiated state ${ }^{68}$ (Fig. 7S), but the total NADH signal was lower in organoids 512 maintained in conventional culture. Furthermore, organoids in the bioreactor demonstrated lower

513 LLS levels suggesting that they experience less oxidative stress than organoids maintained in 514 conventional tissue culture (Fig. 7T). We confirmed with gene expression for retinal genes and 515 observed no significant difference between RtOgs maintained in conventional culture or the 516 bioreactor (Fig. 8A). Because RtOgs expressed similar gene expression profiles after 37 days of 517 on-chip culture to human adult retina, this is promising for future observations optimization to 518 develop mature retinal without segment structures (SFig. 2), which are typically observed under 
519 long term culture conditions ${ }^{69}$. H\&E staining of organoids maintained in the bioreactor or in 520 conventional culture suggested a possible difference in tissue architecture between the two 521 groups. RtOgs maintained in culture may have greater propensity for vacuole formation that 522 correlates anatomically with a greater LLS and oxidative stress (Fig. 8B-E).

\section{CONCLUSION}

525 In this study, we designed and optimized a bioreactor for long term RtOg culture in a low 526 shear stress environment that was also compatible with multimodal imaging. We found that 527 higher flow rates through narrower channels with culture chambers on alternating sides of the 528 perfusion channel enabled optimal and practical concentration uniformity between culture 529 chambers. We subsequently achieved RtOgs culture on a shear stress-free micro-millifluidic 530 bioreactor for 1 month and identified key similarities and differences between RtOgs maintained 531 in either static culture or the bioreactor. We found that: 1) bioreactor cultured RtOgs developed 532 transparent layered surface structure comparable to static cultured ones; 2) the outer surface 533 region of bioreactor cultured RtOgs had comparable free/bound NADH ratio and overall lower 534 long lifetime species (LLS) ratio than static culture RtOgs; 3) RtOgs maintained for 22 days in 535 either the bioreactor or conventional culture expressed significantly higher CRX than that on day 536 98; and 4), there was no significant difference in gene expression between static conventional 537 culture and bioreactor culture. Therefore, the micro-millifluidic bioreactor in this study has 538 demonstrated its potential to sustain RtOgs of equal or better quality than organoids maintained 539 in static culture, while achieving this goal in a low maintenance, sheer stress-free system.

\section{AUTHOR CONTRIBUTIONS}

542 Y.X.: methodology, data curation, formal analysis, investigation, visualization and writing of 543 original draft; M.S.: funding acquisition, project administration, resources, supervision, validation 
544 and manuscript review \& editing; W.T.: conceptualization, project administration, supervision,

545 validation and manuscript review \& editing ; J.W.: investigation, visualization and writing of original

546 draft; A.B.: conceptualization, funding acquisition, methodology, project administration, resources,

547 supervision, validation, visualization, manuscript review \& editing.

548

549 CONFLICTS OF INTEREST

550 There are no conflicts to declare.

551

552 ACKNOWLEDGEMENTS

553 This work was supported by CIRM (\#TRAN1-10995), RPB unrestricted grant to UCI Department

554 of Ophthalmology and ICTS KL2 (\#KL2 TR001416). We thank Dr. Majlinda Lako (Newcastle

555 University, UK.) for donating the CRX GFP hESC cell line, Drs. Michelle Digman (Dept. of BME,

$556 \mathrm{UCI}$ ) and Enrico Gratton (Dept. of $\mathrm{BME}, \mathrm{UCI}$ ) for their help and advice on fluorescence lifetime

557 imaging, and Bryce McLelland (AIVITA Biomedical Inc, California) for instructions on retinal

558 organoids initiation. We thank the Optical Biology Center in UCI for providing microscopic facilities

559 and Stem Cell Research Center Core for qPCR and histology service. We also thank all the

560 undergraduate students that participated in this project: Johnny Garcia (now B.S.), Yuming Ye,

561 Xiyue Yao, Lelin Zhong, Vanna Giang and Mojan Rouhizadeh.

563 REFERENCES

564 1. M. J. Seiler, R. B. Aramant and S. L. Ball, Vision Res, 1999, 39, 2589-2596.

565 2. G. Woch, R. B. Aramant, M. J. Seiler, B. T. Sagdullaev and M. A. McCall, Invest

566 Ophthalmol Vis Sci, 2001, 42, 1669-1676.

567 3. B. T. Sagdullaev, R. B. Aramant, M. J. Seiler, G. Woch and M. A. McCall, Invest

$568 \quad$ Ophthalmol Vis Sci, 2003, 44, 1686-1695. 
4. B. B. Thomas, M. J. Seiler, S. R. Sadda and R. B. Aramant, Exp Eye Res, 2004, 79, 2939.

5. P. B. Yang, M. J. Seiler, R. B. Aramant, F. Yan, M. J. Mahoney, L. M. Kitzes and H. S. Keirstead, Exp Eye Res, 2010, 91, 727-738.

6. M. J. Seiler, R. E. Lin, B. T. McLelland, A. Mathur, B. Lin, J. Sigman, A. T. De Guzman, L. M. Kitzes, R. B. Aramant and B. B. Thomas, Invest Ophthalmol Vis Sci, 2017, 58, 614-630.

7. B. B. Thomas, R. B. Aramant, S. R. Sadda and M. J. Seiler, in Retinal Degenerative Diseases, eds. J. G. Hollyfield, R. E. Anderson and M. M. LaVail, Springer, New York, NY, 2006, pp. 367-376.

8. R. B. Aramant and M. J. Seiler, Prog Retin Eye Res, 2004, 23, 475-494.

9. M. J. Seiler, R. B. Aramant and H. S. Keirstead, Optics and Photonics News, 2008, 19, 37-47.

10. M. J. Seiler and R. B. Aramant, Prog Retin Eye Res, 2012, 31, 661-687.

11. N. D. Radtke, R. B. Aramant, H. M. Petry, P. T. Green, D. J. Pidwell and M. J. Seiler, Am J Ophthalmol, 2008, 146, 172-182.

12. B. A. Tucker, I. H. Park, S. D. Qi, H. J. Klassen, C. Jiang, J. Yao, S. Redenti, G. Q. Daley and M. J. Young, PLOS ONE, 2011, 6, e18992.

13. R. A. Pearson, A. C. Barber, M. Rizzi, C. Hippert, T. Xue, E. L. West, Y. Duran, A. J. Smith, J. Z. Chuang, S. A. Azam, U. F. Luhmann, A. Benucci, C. H. Sung, J. W. Bainbridge, M. Carandini, K. W. Yau, J. C. Sowden and R. R. Ali, Nature, 2012, 485, 99103.

14. M. S. Singh, P. Charbel Issa, R. Butler, C. Martin, D. M. Lipinski, S. Sekaran, A. R. Barnard and R. E. MacLaren, Proc Natl Acad Sci U S A, 2013, 110, 1101-1106.

15. D. A. Lamba, J. Gust and T. A. Reh, Cell Stem Cell, 2009, 4, 73-79.

16. R. E. MacLaren, R. A. Pearson, A. MacNeil, R. H. Douglas, T. E. Salt, M. Akimoto, A. Swaroop, J. C. Sowden and R. R. Ali, Nature, 2006, 444, 203-207.

17. F. C. Mansergh, R. Vawda, S. Millington-Ward, P. F. Kenna, J. Haas, C. Gallagher, J. H. Wilson, P. Humphries, M. Ader and G. J. Farrar, Exp Eye Res, 2010, 91, 500-512.

18. J. A. Thomson, J. Itskovitz-Eldor, S. S. Shapiro, M. A. Waknitz, J. J. Swiergiel, V. S. Marshall and J. M. Jones, science, 1998, 282, 1145-1147.

19. K. Takahashi, K. Tanabe, M. Ohnuki, M. Narita, T. Ichisaka, K. Tomoda and S. Yamanaka, Cell, 2007, 131, 861-872.

20. C. M. Fligor, K. B. Langer, A. Sridhar, Y. Ren, P. K. Shields, M. C. Edler, S. K. Ohlemacher, V. M. Sluch, D. J. Zack and C. Zhang, Scientific reports, 2018, 8, 14520.

21. K. Wahlin, J. Maruotti, S. Sripathi, J. Ball, J. Angueyra, C. Kim, R. Grebe, W. Li, B. Jones and D. Zack, Journal, 2017.

22. T. Nakano, S. Ando, N. Takata, M. Kawada, K. Muguruma, K. Sekiguchi, K. Saito, S. Yonemura, M. Eiraku and Y. Sasai, Cell Stem Cell, 2012, 10, 771-785.

23. B. T. McLelland, B. Lin, A. Mathur, R. B. Aramant, B. B. Thomas, G. Nistor, H. S. Keirstead and M. J. Seiler, Investigative ophthalmology \& visual science, 2018, 59, 2586-2603.

24. J. Assawachananont, M. Mandai, S. Okamoto, C. Yamada, M. Eiraku, S. Yonemura, Y. Sasai and M. Takahashi, Stem cell reports, 2014, 2, 662-674.

25. H. Shirai, M. Mandai, K. Matsushita, A. Kuwahara, S. Yonemura, T. Nakano, J. Assawachananont, T. Kimura, K. Saito and H. Terasaki, Proceedings of the National Academy of Sciences, 2016, 113, E81-E90.

26. S. Llonch, M. Carido and M. Ader, Dev Biol, 2018, 433, 132-143.

27. C. B. Mellough, J. Collin, R. Queen, G. Hilgen, B. Dorgau, D. Zerti, M. Felemban, K. White, E. Sernagor and M. Lako, Stem cells translational medicine, 2019, 8, 694-706. 
619

620

621

622

623

624

625

626

627

628

629

630

631

632

633

634

635

636

637

638

639

640

641

642

643

644

645

646

647

648

649

650

651

652

653

654

655

656

657

658

659

660

661

662

663

664

665

666

667

668

669

28. Z. Ao, H. Cai, D. J. Havert, Z. Wu, Z. Gong, J. M. Beggs, K. Mackie and F. Guo, Anal Chem, 2020, 92, 4630-4638.

29. E. Berger, C. Magliaro, N. Paczia, A. S. Monzel, P. Antony, C. L. Linster, S. Bolognin, A. Ahluwalia and J. C. Schwamborn, Lab Chip, 2018, 18, 3172-3183.

30. M. E. Boutin, C. Hampton, R. Quinn, M. Ferrer and M. J. Song, Adv Exp Med Biol, 2019, 1186, 171-193.

31. T. DiStefano, H. Y. Chen, C. Panebianco, K. D. Kaya, M. J. Brooks, L. Gieser, N. Y. Morgan, T. Pohida and A. Swaroop, Stem cell reports, 2018, 10, 300-313.

32. L. Goto-Silva, N. M. E. Ayad, I. L. Herzog, N. P. Silva, B. Lamien, H. R. B. Orlande, A. da Costa Souza, S. Ribeiro, M. Martins, G. B. Domont, M. Junqueira, F. Tovar-Moll and S. K. Rehen, BMC Dev Biol, 2019, 19, 3.

33. P. Ovando-Roche, E. L. West, M. J. Branch, R. D. Sampson, M. Fernando, P. Munro, A. Georgiadis, M. Rizzi, M. Kloc, A. Naeem, J. Ribeiro, A. J. Smith, A. Gonzalez-Cordero and R. R. Ali, Stem Cell Res Ther, 2018, 9, 156.

34. M. A. Phelan, P. I. Lelkes and A. Swaroop, Stem Cell Investig, 2018, 5, 33.

35. A. Artero Castro, F. J. Rodriguez Jimenez, P. Jendelova and S. Erceg, Stem Cells, 2019, 37, 1496-1504.

36. M. J. Beauchamp, G. P. Nordin and A. T. Woolley, Anal Bioanal Chem, 2017, 409, 43114319.

37. D. Smith, et al. , 2014.

38. E. Berger, C. Magliaro, N. Paczia, A. S. Monzel, P. Antony, C. L. Linster, S. Bolognin, A. Ahluwalia and J. C. Schwamborn, Lab on a Chip, 2018, 18, 3172-3183.

39. B. Sidar, B. R. Jenkins, S. Huang, J. R. Spence, S. T. Walk and J. N. Wilking, Lab Chip, 2019, 19, 3552-3562.

40. D. Qin, Y. Xia and G. M. Whitesides, Nature protocols, 2010, 5, 491-502.

41. J. Collin, C. B. Mellough, B. Dorgau, S. Przyborski, I. Moreno-Gimeno and M. Lako, Stem Cells, 2016, 34, 311-321.

42. X. Zhong, C. Gutierrez, T. Xue, C. Hampton, M. N. Vergara, L.-H. Cao, A. Peters, T. S. Park, E. T. Zambidis and J. S. Meyer, Nature communications, 2014, 5, 4047.

43. M. A. Digman, V. R. Caiolfa, M. Zamai and E. Gratton, Biophys J, 2008, 94, L14-16.

44. S. Ranjit‡, L. Malacridaキ, M. Stakic and E. Gratton.

45. C. Stringari, J. L. Nourse, L. A. Flanagan and E. Gratton, PloS one, 2012, 7, e48014.

46. R. Datta, A. Alfonso-García, R. Cinco and E. Gratton, Sci Rep, 2015, 5, 9848.

47. C.-C. Hong, J.-W. Choi and C. H. Ahn, Lab on a Chip, 2004, 4, 109-113.

48. A. W. Browne, C. Arnesano, N. Harutyunyan, T. Khuu, J. C. Martinez, H. A. Pollack, D. S. Koos, T. C. Lee, S. E. Fraser, R. A. Moats, J. G. Aparicio and D. Cobrinik, Invest Ophthalmol Vis Sci, 2017, 58, 3311-3318.

49. C. Stringari, R. A. Edwards, K. T. Pate, M. L. Waterman, P. J. Donovan and E. Gratton, Scientific reports, 2012, 2, 1-9.

50. B. K. Wright, L. M. Andrews, J. Markham, M. R. Jones, C. Stringari, M. A. Digman and E. Gratton, Biophysical journal, 2012, 103, L7-L9.

51. J. E. Niven and S. B. Laughlin, The Journal of experimental biology, 2008, 211, $1792-$ 1804.

52. R. P. Wolfe and T. Ahsan, Biotechnology and bioengineering, 2013, 110, 1231-1242.

53. S. Regmi, A. Fu and K. Q. Luo, Scientific Reports, 2017, 7, 39975.

54. J. A. Frangos, L. V. Mclntire and S. G. Eskin, Biotechnology and bioengineering, 1988, 32, 1053-1060.

55. P. Ovando-Roche, E. L. West, M. J. Branch, R. D. Sampson, M. Fernando, P. Munro, A. Georgiadis, M. Rizzi, M. Kloc, A. Naeem, J. Ribeiro, A. J. Smith, A. Gonzalez-Cordero and R. R. Ali, Stem Cell Research \& Therapy, 2018, 9, 156.

56. A. Schwartz, Transactions of the American Ophthalmological Society, 1972, 70, 178. 
57. Y. C. Smith, Grande, K. K., Rasmussen, S. B., \& O'Brien, A. D., 2006.

58. A. J. Carterson, Höner zu Bentrup, K., Ott, C. M., Clarke, M. S., Pierson, D. L., Vanderburg, C. R., Buchanan, K. L., Nickerson, C. A., Schurr, M. J., Infection and Immunity, 2005, 73, 1129-1140.

59. R. Salerno-Goncalves, A. Fasano and M. B. Sztein, J Vis Exp, 2016, DOI: $10.3791 / 54148$.

60. B. E. Hjelm, A. N. Berta, C. A. Nickerson, C. J. Arntzen and M. M. Herbst-Kralovetz, Biol Reprod, 2010, 82, 617-627.

61. K. A. Homan, N. Gupta, K. T. Kroll, D. B. Kolesky, M. Skylar-Scott, T. Miyoshi, D. Mau, M. T. Valerius, T. Ferrante, J. V. Bonventre, J. A. Lewis and R. Morizane, Nat Methods, 2019, 16, 255-262.

62. S. D. Ramachandran, K. Schirmer, B. Munst, S. Heinz, S. Ghafoory, S. Wolfl, K. SimonKeller, A. Marx, C. I. Oie, M. P. Ebert, H. Walles, J. Braspenning and K. BreitkopfHeinlein, PLoS One, 2015, 10, e0139345.

63. M. Kasendra, A. Tovaglieri, A. Sontheimer-Phelps, S. Jalili-Firoozinezhad, A. Bein, A. Chalkiadaki, W. Scholl, C. Zhang, H. Rickner, C. A. Richmond, H. Li, D. T. Breault and D. E. Ingber, Sci Rep, 2018, 8, 2871.

64. Y. S. Zhang, J. Aleman, S. R. Shin, T. Kilic, D. Kim, S. A. Mousavi Shaegh, S. Massa, R. Riahi, S. Chae, N. Hu, H. Avci, W. Zhang, A. Silvestri, A. Sanati Nezhad, A. Manbohi, F. De Ferrari, A. Polini, G. Calzone, N. Shaikh, P. Alerasool, E. Budina, J. Kang, N. Bhise, J. Ribas, A. Pourmand, A. Skardal, T. Shupe, C. E. Bishop, M. R. Dokmeci, A. Atala and A. Khademhosseini, Proc Natl Acad Sci U S A, 2017, 114, E2293-E2302.

65. G. Mattei, Giusti, Serena, Ahluwalia, Arti, 2014, 2, 548-569.

66. N. P. Macdonald, J. M. Cabot, P. Smejkal, R. M. Guijt, B. Paull and M. C. Breadmore, Analytical Chemistry, 2017, 89, 3858-3866.

67. K. P. Archberger, C.; Haderspeck, J.; Bolz, S.; Rogal, J.; Chuchuy, J.; Nikolova, M.; Cora, V.; Antowiak, L.; Haq, W.; Shen, N.; Schenke-Layland, K.; Ueffing, M.; Liebau, S.; Loskill, P. , eLife 2019, DOI: 10.7554/eLife.46188.

68. R. Datta, C. Heylman, S. C. George and E. Gratton, Biomedical optics express, 2016, 7, 1690-1701.

69. K. Achberger, J. C. Haderspeck, A. Kleger and S. Liebau, Adv Drug Deliv Rev, 2019, $140,33-50$. 